\title{
Modelo de Gestión Educativa en el fortalecimiento de la calidad de la tutoría del colegio Francisco Izquierdo Ríos, Morales - 2020
}

\author{
Edwin Rengifo Guerra \\ edwinrengifoguerra@gmail.com \\ Universidad César Vallejo
}

\section{RESUMEN}

La presente investigación tiene como objetivo Proponer que la calidad de la tutoría en la Educación Básica regular del colegio Francisco Izquierdo Ríos, Morales, San Martín, 2020, donde presente la metodología es de tipo Aplicado, debido a que se tuvo un enfoque donde las teorías se tienen en cuenta para el desarrollo de nuevos conocimientos y que son tratados como tal. Llegando a las siguientes conclusiones. El papel que tiene las instituciones de educación básica regular para apoyar el tutoría es muy grande ya que deben enfrentar y participar en los diversos problemas académicos que afectan a los estudiantes aportando vivencias y experiencias del profesorado para superarlos y muy específicamente en el colegio Francisco Izquierdo Ríos, es por esto la importancia que tiene la gestión educativa dentro de los procesos de la institución como factor de calidad fortaleciendo las políticas existentes. 5.2. Con los resultados de esta investigación se percibir que la mayoría de los entrevistados tienen conocimiento de la gestión educativa y la importancia de la tutoría, sin embargo, un 33\% de los encuestados consideran que debería de incrementarse el nivel de compromiso de la institución como eje rector para la consolidación de la gestión educativa

Palabras clave: Gestión educativa, calidad de tutoría, institución educativa. 


\title{
Educational Management Model in strengthening the quality of tutoring at the Francisco Izquierdo Ríos, Morales school - 2020
}

\begin{abstract}
The present research aims to propose that the quality of the tutoring in the regular Basic Education of the Francisco Izquierdo Ríos school, Morales, San Martín, 2020, where the methodology is of Applied type, because it had an approach where the theories they are taken into account for the development of new knowledge and are treated as such. Arriving at the following conclusions. The role of regular basic education institutions to support tutoring is very large since they must face and participate in the various academic problems that affect students, contributing experiences and experiences of the teaching staff to overcome them and very specifically in the Francisco Izquierdo Ríos school This is why the importance of educational management within the institution's processes as a quality factor, strengthening existing policies. 5.2. With the results of this research, it can be seen that the majority of the interviewees have knowledge of educational management and the importance of tutoring, however, $33 \%$ of the respondents consider that the level of commitment of the institution should be increased as an axis rector for the consolidation of educational management
\end{abstract}

Keywords: Educational management, quality of tutoring, educational institution

Artículo recibido: 03 nov. 2020

Aceptado para publicación: 07 dic. 2020

Correspondencia edwinrengifoguerra@gmail.com

Conflictos de Interés: Ninguna que declarar 


\section{INTRODUCCIÓN}

En la actualidad, como lo menciona Muñoz (2016) se puede determinar que la falta de valores en la educación se convirtió en uno de los puntos más importantes por resolver dentro de la sociedad, ya que son la base problemas que se vinculan inclusive con el rendimiento escolar, repercutiendo en responsabilidades, ausencia de aspiraciones. Asimismo, a pesar de que se encuentre con los mejores docentes o en la mejor escuela, si esta brinda conocimientos sin valores, no generará un gran desarrollo escolar. (p. 37)

Así mismo Rico (2018) consolida que a nivel global la gestión educativa tiene que orientada a que se optimice el contenido de información del docente de enseñanza a nivel superior. Por ejemplo, en Colombia la gestión educativa planteada por su gobierno es entendida como una parte relevante dentro de la educación, principalmente considerando el rol que cumple en la optimización de las etapas metódicas que logran que el docente se forme. De esta manera, se debe partir por tener un concepto claro del término gestión, así se lograría tener un panorama amplio de la gestión educativa y todo lo que comprende, de esta manera finalmente se logra un análisis correcto de esta dentro de una institución en la educación básica regular.

Por ello, en Nicaragua en base a lo que menciona Fiallo, M. (2016, p. 21) la ausencia de valores en las instituciones educativas ha afectado directamente en el comportamiento del menor o joven, por lo que la educación de Nicaragua se ha convertido en una educación de tan solo una cara, incluso aplicándose de manera ineficiente con respecto al artículo 116 de la Constitución de dicho país y según el cuarto artículo de la ley general de la educación.

En España, Soto (2017), uno de los ganadores al premio Educa Abanca, en el año 2017 hizo mención del problema de los valores en las escuelas y es que según menciona el autor, muchas personas consideran que la escuela sólo brinda educación cognitiva, dejando de lado a los valores de lado de los cuales se deben hacer cargo lo padres, pero el autor se niega aceptar ello, debido a que la educación es completa y va de la mano con los valores, de manera que se enseñan materias no sólo para producir sino para convivir en la sociedad. Por lo que la importancia de los valores en los centros educativos radica que en base a ellos se encuentra la formación de los estudiantes de manera integral, debiéndose los centros educativos empoderarse, colocándose al mismo nivel que las materias. (p. 211)

En Bruselas Saez (2015) refiere que el 20\% de escolares abandona de manera prematura la escuela, debido a la ausencia de valores tanto en la educación escolar, como en sus propios 
hogares, es por ello que surge la necesidad de una estrategia adecuada que brinde resultados a mediano y largo plazo, mediante manejo de técnicas de estudio para los estudiantes. (p. 42).

Entonces podemos indicar que el fracaso escolar se debe a que no se brinda una educación de calidad, definida por la no aplicación de reformas estructuradas en el sistema educativo. Razones por las cuales existe un direccionamiento hacia solucionar la ausencia de valores en la educación peruana mediante la tutoría, por ende, diversos estudios que se han realizado a determinar la eficiencia o eficacia de esta materia, permitiendo determinar propuestas que puedan acelerar el rendimiento y cultivar valores y orientación en todos los ejes de la persona, (Flores, 2016, p. 18).

En la I.E. Francisco Izquierdo Ríos ubicada en el distrito de Morales existe una problemática latente en cuanto a la ausencia de tutoría, teniendo consecuencias en el rendimiento académico. Asimismo, la desorientación en educación sexual trae consigo casos de embarazo precoz, frustrando el proyecto de vida del estudiante; además problemas conductuales, por apartarse de la práctica de valores manifestados en comportamientos inadecuados, siendo una posible causante la ausencia de un trabajo sostenido en el área de Tutoría dentro de la institución, convirtiéndola en una posible solución, permitiendo así mejorar los resultados que los estudiantes obtienen en términos de aprendizaje en los diversos ejes de su desarrollo. Por otro lado, es necesaria una mejora en la gestión educativa y en las diferentes áreas lectivas; permitiendo generar planes más efectivos que puedan satisfacer la latente demanda educativa. Con ello el planteamiento acorde a la actualidad y a los problemas sociales que enfrenta el Perú, donde existan contingencias como tales. Es por ello que es necesario tomar como referencia la siguiente interrogante ¿Qué estructura tendrá el modelo de gestión educativa en aras de fortalecer las condiciones de la tutoría en términos de calidad en la Educación Básica regular del colegio Francisco Izquierdo Ríos, Morales, San Martín, 2020?

Es así como la investigación se justifica en teorías iniciales como parte de la gestión pedagógica abordado por Frigerio et al. (1992), quienes dimensionaron la gestión pedagógica en cuatro principales ejes, por lo que a partir de este postulado es que en América Latina de han tomado éstas dimensiones para el manejo de esta variable, así mismo se hizo uso de información que se encuentra estipulado en el Ministerio de Educación, como parte de la ley de la educación. El estudio pretende abarca metodológicamente conforme 
al autor Hurtado (2014), que enfoca un claro diseño útil en dar respuesta a la problemática que se pretende abordar, en base al método científico, qué como se conoce parte de observar directamente el fenómeno (problema) a estudiar, para llegar a tener conclusiones razonables con los planteamientos previos y con fundamento. A través del estudio investigativo se plasma una posible solución enfocada en una institución que tiene en común una problemática con otras instituciones de similares características, por ello la investigación repercutirá en las discusiones de próximos estudios con características similares en su problemática.

Por lo expuesto, se plantea como objetivo general lo siguiente: Proponer que la calidad de la tutoría en la Educación Básica regular del colegio Francisco Izquierdo Ríos, Morales, San Martín, 2020, se mejore a través de un modelo de gestión educativa fundamentado en la teoría de Frigerio, Poggi, Tiramonti y Aguerrondo, que a su vez contenga los siguientes objetivos específicos: i) Caracterizar la calidad de la tutoría en la Educación Básica Regular del colegio Francisco Izquierdo Ríos, Morales, San Martín, 2020. ii) Caracterizar la calidad de la gestión educativa en la Educación Básica Regular del colegio Francisco Izquierdo Ríos, Morales, San Martín, 2020 iii) Diseñar el modelo de gestión educativa para fortalecer la calidad de la tutoría en la Educación Básica Regular del colegio Francisco Izquierdo Ríos, Morales, San Martín, 2020, fundamentado en la teoría de Frigerio, Poggi, Tiramonti y Aguerrondo. Iv) Validar el modelo de gestión propuesto a través de juicio de expertos. Obteniendo la siguiente hipótesis: El modelo de gestión educativa basado en la teoría de Frigerio, Poggi, Tiramonti y Aguerrondo fortalecerá la calidad de aprendizaje en la Educación Básica Alternativa del colegio Francisco Izquierdo Ríos, Morales, San Martín, 2020 ,

\section{MARCO TEÓRICO}

De acuerdo a lo tomado como problemática el desempeño investigativo toma como concisa la búsqueda de adecuadas investigaciones que valoren y generen un mayor énfasis en la problemática internacional como lo tomado en la investigación de Portugal (2018) que lleva como título "La Gestión Educativa: Una Visión hacia la Formación Docente" de la Universidad Santo Tomás (artículo científico) y plantea un objetivo general de diseñar estrategias de gestión educativa a través de la motricidad de la personas tomando en cuenta referencias aplicadas en la ciudad de Barcelona y donde muestra las siguientes conclusiones. Se debe resaltar la importancia de que exista la posibilidad de cambiar el 
enfoque "cultural" que los docentes futuros poseen e inculcan a sus educandos venideros, básicamente en la forma en como ellos asumen la gestión, calidad educativa y el acervo de conocimientos, como parte de un conjunto de partes que pueden son variantes desde la función que cada uno realiza. Es cierto que hay una tendencia a querer que la educación sea homogénea, pero resulta innegable la heterogeneidad que se encuentra constante y viva en el entorno educativo de cada realidad.

Por otro lado, García (2018) en el artículo científico "Gestión escolar y calidad educativa" tiene como objetivo evidenciar los factores asociados a la gestión educativa dentro de la realidad de la población mexicana, para lo cual se toma como punto de partida el año 2015, momento en el que se da una reforma en la educación que busca una orientación diferente del proceso de enseñanza. Para tales fines se exponen los factores necesarios para la consecución de resultados según lo que la reforma plantea. Sin embargo, también se plasman preguntas que en la búsqueda de darles respuesta promuevan mejoras en las políticas educativas, tanto instituciones educativas de nivel media como superior.

Así mismo Sánchez (2016) muestra un artículo científico titulado "La gestión educativa como factor de calidad en una universidad intercultural" de la Universidad Autónoma Indígena de México tomando como objetivo principal resolver el gran problema que enfrenta la Universidad Autónoma Indígena de México (UAIM) para obtener altos estándares de calidad conforme a lo que solicita la Subsecretaria de Educación Superior, mostrando así problemas en la gestión educativa de esta institución, es así que mediante este artículo se cuestiona y analiza las cuatro dimensiones de la gestión educativa: organizacional, pedagógica, administrativa y comunitaria; adicionalmente el engranaje de las mencionadas con la finalidad de que el funcionamiento de la institución mejore.

En parte por el campo de la tutoría se tiene que Badillo (2016) en su artículo científico "La tutoría como estrategia viable de mejoramiento de la calidad de la educación superior. Reflexiones en torno al curso" del Instituto de Investigaciones en Educación. Analiza el impacto del curso en los enormes escenarios de mejora que ofrece la tutoría en las instituciones educativas de nivel superior, ya que al tener un vasto conocimiento de los estudiantes, se optimiza el escenario en el que se diseñan e implementan las estrategias de atención, de esta manera se logran niveles significativamente más grandes de calidad de la enseñanza, sucesivamente el poder de la institucional de no perder alumnos también mejora, finalmente es posible formar no solo mayor cantidad de profesionales, si no 
también y de mejor calidad. De esta manera, se analizó otros diferentes aspectos que debe considerar la tutoría, ya que existen varias instituciones en las que este curso solo se limita a la atención de la parte escolarizada del alumno y dejan de lado otros aspectos posibles a intervenir como la situación social y económica que atraviesa, y para finalizar, pero no por ello menos importante sus condiciones afectivas y de salud. En este sentido, es claro que el tutor no debe actuar como un psicólogo (debido a que es común que no tenga la capacitación necesaria para realizar ello), tampoco le compete tomar decisiones por el estudiante, sin embargo, la importancia radica en que el tutor se encuentre atento a los factores que influyen en el rendimiento del alumno. Esta atención debe desembocar en que los casos que necesiten atención especializada sean derivados a profesionales específicos, en busca de favorecer el desarrollo integral de cada estudiante y evitando que las situaciones problemáticas de alrededor afecten su aprendizaje negativamente.

Como referencia enigmática la investigación de López (2017) en el artículo científico titulado "Estudio Longitudinal Sobre Tutoría Académica Flexible En La Universidad" Afirma que es preciso destacar que el reconocimiento institucional de la tutoría electrónica ha supuesto no solamente un incentivo real (la reducción del 50\% del horario presencial de tutoría) sino también ha constituido un mecanismo de difusión de este modelo flexible de acción tutorial que algunos profesores desconocían. Es así con el propósito de llevar a un nivel de mayor profundidad en la investigación se tiene que existe estudios anteriores a ello que determinan el comportamiento de las variables.

En complementación de la información se tiene que Coronado (2017) en el artículo científico "Tutoría y orientación educativa para mejorar las habilidades sociales en los estudiantes de la institución educativa Multigrado $N^{\circ}$ 16165, Huaranguillo - San José del Alto - Jaén". Universidad de Cajamarca. Perú. Tiene un tipo de investigación pre experimental, empleando una muestra de 47 estudiantes de la institución educativa número 16165, a la que se le realizó la técnica de pre test y post test, permitiendo concluir que mediante la aplicación de tutoría se ha mejorado las habilidades de interacción social en un $89.4 \%$ además las conversacionales en un $72.3 \%$, permitiendo determinar un programa eficaz para fortalecer habilidades de los estudiantes al interactuar socialmente y al conversar. La investigación es referencial para la segunda variable ya que muestra la efectividad de cualquier plan sobre orientación estudiantil con respecto a la materia de Tutoría, de la cual se pude tener información para el marco teórico y futuras discusiones. 
Miranda (2015) en su artículo "El sistema de tutoría en el desarrollo integral de los estudiantes de la Universidad César Vallejo Tarapoto”. Universidad César Vallejo. Tarapoto, Perú. La investigación tuvo un diseño explicativo causal de enfoque mixto, se empleó la técnica de la encuesta para el análisis de los datos, dirigida a los estudiantes de la Universidad permitiendo concluir que existe una carencia en el sistema de tutoría de $6.85 \%$, por lo que la propuesta contempla un sistema direccionado hacia las carencias determinadas por la encuesta por lo que es necesaria la participación más activa del área mediante un proceso de orientación continua. La investigación es un referente ya que permite analizar información con respecto a la variable dependiente, por lo que su instrumento es de gran ayuda para el estudio.

Con ello definimos que el proceso de gestión educativa se encuentra en un nivel de investigación amplio pero que no fue categorizado adecuadamente de acuerdo a la tutoría y se inicia con una búsqueda exhaustiva de teorías que respalden a las variables de estudios iniciando con la conceptualización de gestión donde según UNESCO (2013), el término gestión se entiende como el consolidado de ideas estructuradas, con el objetivo de poder generar objetivos estratégicos y poder alcanzarlos, esta manera de definirla ha sido producto de la diferentes autores quienes abordaron este campo y es a inicios de siglo XX que Weber determinó la organización del trabajo, como un procesos que busca un ajuste en los diferentes medios para poder alcanzar los fines propuestos, asimismo Taylor desarrolló la idea de gestión científica, la cual fue complementada por Fayol, por lo que ambos fueron considerados padres de la administración.

De acuerdo a la UNESCO (2013), definir a la gestión se torna muy complejo, por lo que mediante el enfoque en la movilización de los recursos dentro de una institución es tomada como el conjunto de condiciones indispensables para lograr obtener una adecuada relación de las estrategias, estructura, el estilo, sistemas, las personas, capacidades y los objetivos organzacionales calificados como superiores, por lo que es tomada como aquella capacidad que se tiene dentro de una institución para poder articular lo que se tenga a disposición como recurso, para así obtener lo que se ha propuesto.

Por su parte, Cassasús (2014), hizo mención acerca de los inicios de la gestión educativa la cual tuvo sus inicios en el Reino Unido durante los setenta, trasladándose a Latinoamérica en los años 80, en la búsqueda de poner en práctica los pilares de la gestión direccionados a la educación, presentando como objetivo estudiar la organización de la labor en dicha área 
determinada por las teorías generales. Por otro lado no se trata de una rama por separado se trata del manejo de principios tanto de la gestión como de la educación, por lo que se habla de una disciplina que se aplica en un contexto específico donde toma acción.

Para Cassasús (2014): “La gestión educativa es considerada como una disciplina en la cual interactúa teoría, política y pragmática” (p. 97).

De acuerdo a Cassasús (2014 la gestión educativa cuenta con cuatro dimensiones claramente identificadas. Además estas dimensiones son tomadas por los autores a partir del denominado campo institucional, donde diversos elementos interactúan en un momento dado, este debe ser siempre dinámico, restructurándose en modificándose de manera permanente, dentro de éstas se pueden determinar las siguientes:

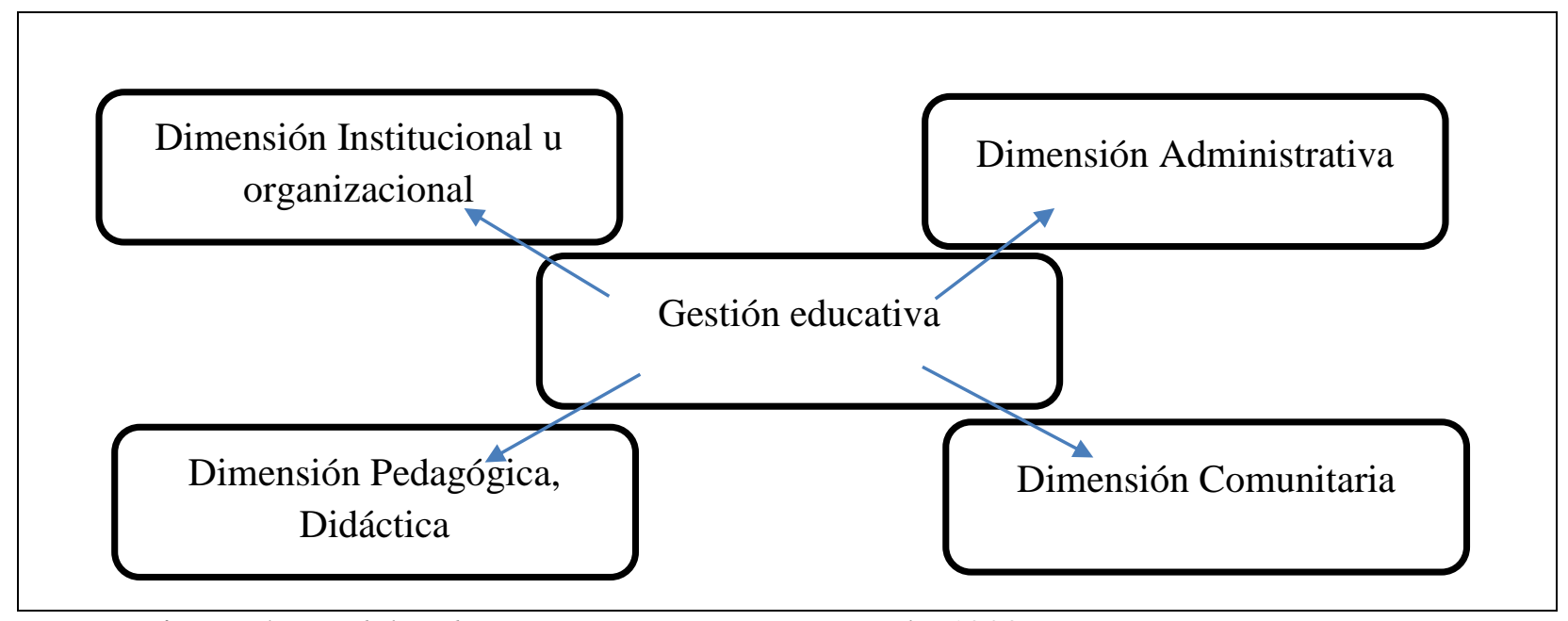

Figura 1: Modelos de gestión según Frigerio et al. (1992).

Elaboración propia Fuente: Frigerio et al. (1992).

Así tenemos, que la dimensión institucional u organizacional, es considerada como, el conglomerado de características estructurales determinan la forma en que funciona la gestión en sí, ya que tales características están presentes en cada establecimiento educativo, tomándose en cuenta temas relativos a la estructura formal, por ejemplo, se consideran los organigramas, división del trabajo, distribución de tareas, múltiples objetivos, empleo del tiempo y espacios, canales de comunicación. Además se debe tomar en cuenta aspectos relativos a la estructuras informales, tomándose en cuenta participantes que cumplen un rol institucional desarrollando actividades dentro de las estructuras formales, (Frigerio et al., 1992).

Asimismo, la dimensión administrativa, está referida a prácticas de gobierno, ya que un administrador realiza actividades que generan estrategias mediante la planificación. En la 
consecución de estos fines considera recursos humanos, recursos financieros y el factor tiempo, además debe tener bajo control el desarrollo consecutivo de acciones correctivas que permitan mejoras, La administración debe convertirse en el objeto que facilita la gestión actual y a futuro, considerando los objetivos trazados. Para ello tener dominio sobre la información en cuanto a conocimiento y disponibilidad, es un aspecto netamente importante, por ende la información es significativa y aporta a la en la correcta toma de decisiones intra institucionales, (Frigerio et al., 1992)

En la dimensión pedagógica, que considera las metodologías de enseñanza, es decir la didáctica referida a las acciones que caracterizan a una institución educativa, genera una diferencia entre otras instituciones; siendo su principal eje constituido por lazos que los actores construyen entre los modelos didácticos y el conocimiento, es por lo mencionado que esto refleja puntos fundamentales a mostrar en esta dimensión modalidades de enseñanza junto a las teorías de enseñanza y de aprendizaje que se encuentran en base a las actividades continuas de los docentes, utilizadas como parte de la práctica día a día, el saber en la esencia de su valor y significado, reglas para evaluar procesos y los principales resultado que se muestran.

En la dimensión comunitaria, referida a acciones que fomentan que los diferentes actores participen en la toma de decisiones, así como en toda actividad que compete al establecimiento y la interacción con los representantes de entorno que le corresponde. Además, tiene que ver con la manera en que las instituciones toman como suyas las demandas, los reclamos y problemas que recibe en su ámbito. Es importante que se separen asuntos que formarán parte de la responsabilidad del establecimiento escolar de algunas cuestiones que podrán ser luego reconsideradas por otras instituciones. (Frigerio et al., 1992).

Asimismo, referente a la dimensión institucional: Trata sobre las maneras en que se organiza la institución, también enfoca las características de su estructura, lugares jurisdiccionales disponibles y las respectivas responsabilidades. En esta dimensión se consideran las características de las relaciones, además de las normas estrictamente especificadas y las que no (Domínguez, 2016, p. 198)

En cuanto a la dimensión Administrativa: Comprende la administración de los recursos económicos, dentro de los cuales encontramos los recursos humanos y materiales, 
procedimientos técnicos, recurso tiempo, higiene y seguridad que corresponde en las realizaciones de actividades conforme a la normatividad.

El manejo de sistemas de información y otros tipos de datos. Se asegura de que cada uno de los proceso se realicen conforme a la normatividad, lo cual requiere vigilancia. (Domínguez, 2016, p.205)

Además en la dimensión Pedagógica: El abanico de posibilidades de metodologías en la educación. El proceso implica en primer lugar la planificación, conforme a lo que se trace en esta primera parte se considera una evaluación y según las circunstancias y resultados una posible certificación. La manera en que las actividades pedagógicas constantes se desarrollan es otro punto a considerar. Por otro lado, se debe procurar que los docentes se actualicen de forma constante para el desarrollo de su actividad de la manera más eficiente, la financiación de lo mencionado, puede marcar las diferencias entre instituciones. (Domínguez, 2016, p.211)

Finalmente en la dimensión Comunitaria Es claro que la gestión se orienta al beneficio de las comunidades, por lo cual los resultados deben dar respuesta a las carencias de la misma. La importancia de marcar relaciones fuertes entre la escuela y lo que la rodea no debe dejarse pasar. En este camino es fundamental la comunicación con los familiares tutores, en su mayoría madres y padres (Domínguez, 2016, p.214). Un factor a considerar en cuanto a la localidad, forma en la que se organizan, asimismo planificar y mostrar los canales de apoyo.

\section{Procedimientos de cada Dimensión}

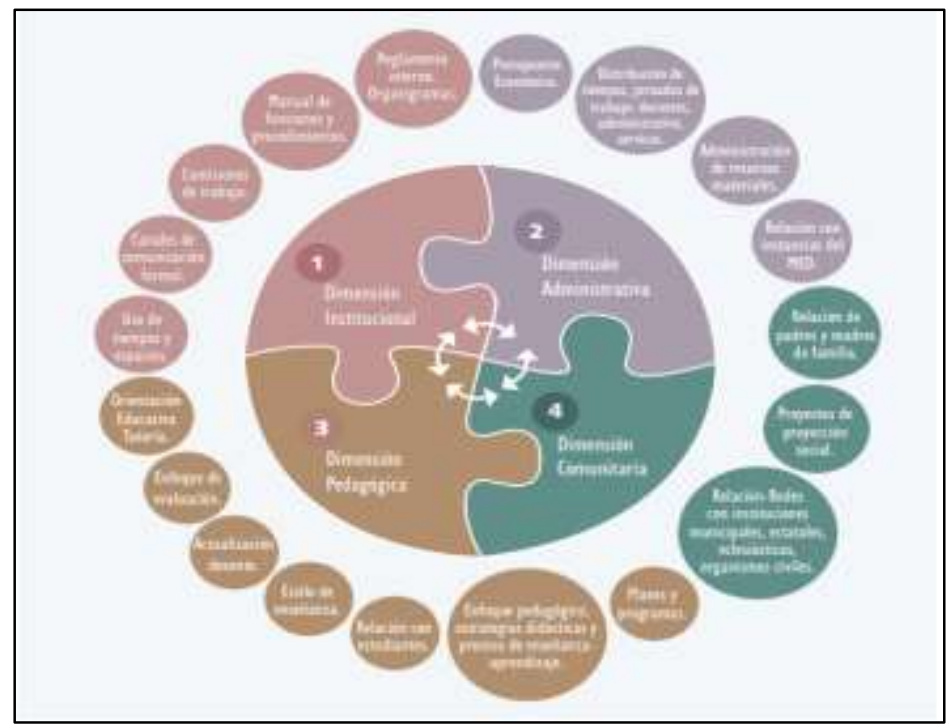

Figura 2: Procedimientos por dimensiones (UNESCO, 2013).

Fuente: (UNESCO, 2013). 
De acuerdo a la figura anterior cada dimensión cuenta con diferentes procedimientos los cuales obedecen a las prácticas que conlleven a una buena gestión educativa, (UNESCO, 2011).

Domínguez. (2016), hizo mención que toda gestión de cualquier tipo de institución, debe tener en cuenta el ciclo de Deming, ya que todo proceso se encuentra direccionado a la búsqueda de la calidad.

Asimismo UNESCO (2013), determina los elementos del ciclo de Deming en la gestión educativa, mismos que se encuentran plasmados en autores con Chiavenato, Koonts y Amarate, teniendo cuatro puntos fundamentales:

El primero lugar se encuentra la dimensión Planificar, referido al proceso sistémico, abierto y continuo, que permite determinar formas de actuación que se puedan aplicar a la educación; además se considera como el proceso de poder tener listo un grupo de decisiones para una actuación en tiempo posteriores, permitiendo la consecución objetivos, (UNESCO, 2013, p.69). Seguido de la dimensión Hacer que representada que no es más que el primer momento de ejecutar todo lo planificado, implica hacer efectivos los procedimientos de gestión, mediante el cual se facilita que los docentes logren integrarse y coordinen al momento en el que realizan sus actividades, las características mencionadas deben darse en la interacción con tutores familiares de los estudiantes y ellos mismos, de la misma manera el uso de recursos dirigidos al desarrollo de los proyectos y programas, involucrando el reparto de las tareas y funciones mediante la facultad de mando y muestras de asumir responsabilidad además de una representación clara de las relaciones entre los que participan y lo demás que los rodea y constituye, (UNESCO, 2013, p.78). Por otro lado, está la dimensión de Verificar que conlleva la etapa de verificación se evalúa que la ejecución pueda responder a lo estipulado, sin perder de vista que brinde las circunstancias óptimas para que se verifique que se cumpla la organización o esquema de responsabilidades y la división del trabajo que fue diseñado con el fin de llegar a las metas y objetivos planteados en las áreas que se han considerado en la planificación. Asimismo, permite generar ajustes en la asignación de los recursos. (UNESCO, 2013, p.82). Y por último esta la dimensión de Actuar, en esta etapa se considera una segunda ejecución, pero con la diferencia que se debe tomar en cuenta lo evaluado, por lo que conlleva a tomar en cuenta los reajustes generados en el proceso para poder conservar metas institucionales. (UNESCO, 2013, p.89). Con ello se define que la enseñanza - Tutoría Ravello, Martínez 
\& Delgado (2015), definieron el término enseñanza como aquella modalidad de orientación de tipo pedagógico por lo que se entiende como aquel servicio que acompaña a lo cognitivo socio afectivo y pedagógico de aprendices, se encuentra dentro del desarrollo conforme a la currícula aportando al logro del aprendizaje, de manera que comprenda todos los aspectos desde un enfoque del desarrollo humano. Es así que, mediante el empleo de la enseñanza dentro de instituciones educativas se garantiza que los estudiantes puedan tener orientación que les corresponde por derecho según lo estipula la ley número 28044, en su artículo 53 inciso a, siendo una medida preventiva de problemas que pueden aparecer en los menores. (Ley General de la Educación $\mathrm{N}^{\circ}$ 28044, 2014). Por lo tanto su aplicación depende directamente del aporte y compromiso de todas los que se encuentran involucrados en la comunidad educativa, mediante profesores, tutores familiares, personal administrativo y estudiantes, por lo que forma gran relevancia dentro del proceso conjunto de aprendizaje, siendo obligatoria una hora dentro de del horario. (Ravello, Martínez \& Delgado, 2015).

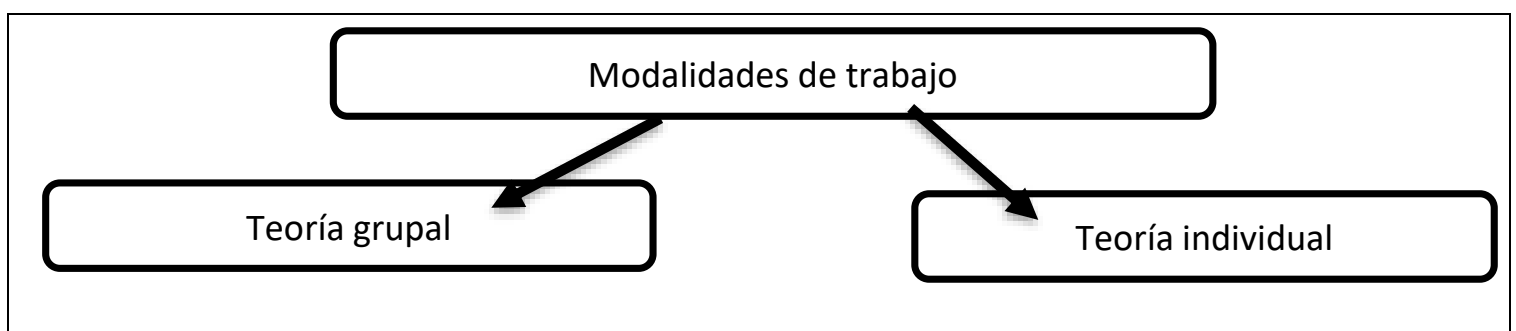

Figura 3: modalidades de trabajo en enseñanza

Elaboración propia. Fuente: (Ravello, Martínez \& Delgado, 2015).

De la figura anterior a lo que respecta la enseñanza grupal no es más que el trabajo o aplicación de la orientación a un conjunto de alumno siendo el total de estudiantes del aula, por lo que en esta área el docente podrá interactuar y orientar a los jóvenes respondiendo dudas e inquietudes, apoyando así al crecimiento del alumno. Este tipo de trabajo presenta flexibilidad por lo que está en constante cambio de acuerdo a lo determinante en la unidad grupal, (Ravello, Martínez \& Delgado, 2015).

En cuanto a la teoría individual cuyo mismo nombre lo dice no es más que la comunicación directa entre alumno y tutor de manera directa, esto sucede cuando en el caso ya no es posible tratar de manera grupal el problema u orientación, (Ravello, Martínez \& Delgado, 2015). 
Pilares de la enseñanza educativa

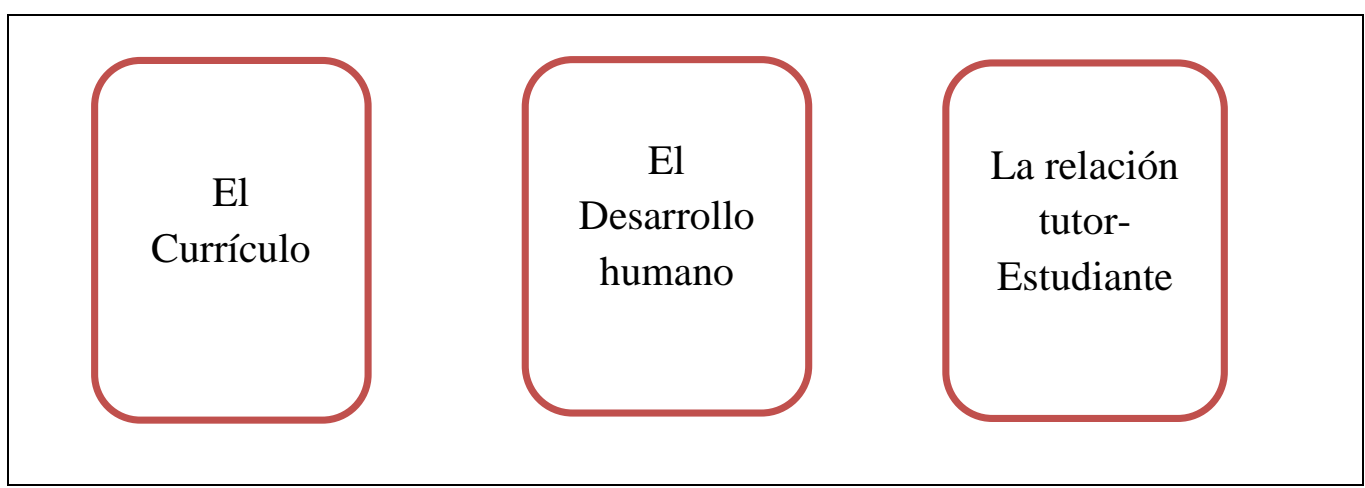

Figura 4: Pilares de la enseñanza educativa

Elaboración propia. Fuente: (Ravello, Martínez \& Delgado, 2015).

De acuerdo a lo anterior en el currículo se puede observar el grupo de elementos que conforman la intencionalidad educativa señalando temáticas a aprender importantes que todo estudiante debe lograr cumplir en etapas según el nivel educativo que vaya escalando, sin importar el lugar, región u otra subdivisión del territorio, buscando la calidad y el equilibrio en todo lo que compete a educación, por otro lado el desarrollo humano refiere al proceso de desarrollo desde la etapa de antes del nacimiento hasta el fin de la vida, tal proceso comprende un conjunto de condiciones y elementos que originan un conjunto sucesivo de variaciones cuantitativas y cualitativas, teniendo un enfoque en la complejidad de los procesos de construcción e interacción mutua en la que interactúa personas y el entorno que los rodea, teniendo un contenido netamente ético. La relación es determinada con un componente fundamental en los procesos de desarrollo, por lo que los estudiantes, sin excepción, requieren de un adulto acompañante y orientador en su formación, con la finalidad de poder generar un desarrollo óptimo, por lo que la relación es por excelencia el que otorga la calidad educativa. Con ello se puede afirmar sobre las Áreas de Enseñanza, donde Ravello, Martínez\& Delgado (2015), Entre las principales se cuentan con las siguientes: Persona social: permite el equilibrio de su personalidad, desarrollando una personalidad sana que permita una buena manera de desenvolverse en su ambiente social. En este ambiente Académico mediante ésta área se asesora y conduce a los estudiantes con la finalidad de que logren alcanzar un rendimiento alto en actividades que realicen dentro de su programación educativa y puedan superar dificultades, Consiguiente del indicador vocacional: Apoya al estudiante para que tomen decisiones fundamentadas sobre el oficio, ocupación o profesión que deseen seguir, considerando lo establecido en su proyecto de vida, el cual debe responder a sus condiciones y elementos que más lo definen, las 
probabilidades de acceso que tengan en términos económicos, geográficos y otros, en el aspecto personal, así como el del su respectivo entorno. Salud mental y corporal: Fomenta el adoptar estilos de vida óptimos y saludables. Ayuda social: direcciona al estudiante a que participe con la comunidad trazándose objetivos que busquen el bienestar de esta y por último la Cultura y actualidad: fomenta que la cultura se valore por parte del estudiante, generando reflexión sobre la actualidad, promoviendo el involucramiento en contextos a nivel local, a nivel de región, de nación y mundial. Área de convivencia y disciplina escolar: Pretende aportar en la fijación de relaciones demográficas y armónicas, todo dentro de lo que comprende normas de convivencia, principalmente el respeto. (p. 147) Llevando a si a los Objetivos de la enseñanza que conlleva en generar una compañía constante en el ámbito socio afectivo, además del tema cognitivo, con el objeto de lograr sumar esfuerzos y resultados a una formación completa y coherente en sus diferentes aspectos, orientando en el desarrollo del mismo evitando y previniendo futuros problemas en el alumno. Características de la Enseñanza de acuerdo a lo abordado por Delgado et al. (2014) entre las características de la enseñanza se encuentran las que a continuación se mencionan y es formativa, ya que a través de la enseñanza se orienta a los alumnos a poder obtener capacidades, habilidades, competencias, actitudes y especialmente valores que hacen posible que se haga frente a dificultades y exigencias que se dan a lo largo del transcurso de su desarrollo. Se puede decir que es preventiva, ya que pretenden que se vayan creciendo los determinantes que protegen y disminuyen los riesgos, por lo que intenta actuar con anticipación a las situaciones problemáticas, a través de estrategias que el estudiante tendrá la oportunidad de utilizar. Por otro lado, personalizada, ya que se brinda la atención para todos los estudiantes de forma particular, el crecimiento de cada estudiante es un proceso complicado ya que se encuentra patrones típicos y que son fácilmente posibles de prevenir en su persona sin dejar de lado los patrones hereditarios, sociales, ambientales y culturales, los cuales hacen de un estudiante único. Y en consecuencia, permanente, ya que el estudiante de la institución tiene apoyo para poder manejar cualquier situación propia en su proceso de desarrollo.

Integral, Debido a que mediante la enseñanza se fomenta la formación de estudiantes en sus diferentes dimensiones como es el caso de lo afectivo, cognitivo, físico, moral y social. Por ello se afirma inclusiva, porque todos los estudiantes sin dejar de lado ninguno, deben recibir guía y disciplina en el seguimiento del proceso, debido a que todas las secciones 
cuentan con enseñanza para ello. Consiguiente recuperadora, de darse el caso que surja alguna dificultad en los estudiantes, el vínculo de apoyo y soporte que es brindada por el tutor mitigan los efectos, por otro lado, hace posible detectarlo a manera temprana para su oportuna intervención, permitiendo una disminución de complicaciones por ultimo no es terapéutica, ya que no tiene como esencia brindar terapia a los estudiantes con diversos problemas, ya que el tutor no pretende sustituir al especialista psicológico o profesional de la salud mental, más bien éste actúa como una de apoyo en la institución ya que, de lograr que se realice la enseñanza de forma terapéutica, las oportunidades de atención serían limitadas. (p. 158). Con ello las funciones de la dirección de enseñanza educativa Según el Ministerio de Educación, MINEDU (2014), la dirección de Enseñanza tiene la responsabilidad de establecer normas, planificar coordinar, dirigir, ejecutar, evaluar, monitorear y difundir, estrategias o políticas de enseñanza y orientación educativa, este depende directamente del viceministro de gestión pedagógica. Donde influyen los modelos de gestión de la calidad educativa o de enseñanza - aprendizaje actual en el mundo y en el Perú. Argyris y Schón (1978), citado por (UNESCO, 2013), lo determina como la capacidad que se tiene para poder articular una representación mental de los miembros que pertenecen o se encuentran involucrados en una organización. Por ello diversas visiones involucran la gestión con el aprendizaje continuo, en en como generan valores y amplían la visión hacia la comunidad, además de las representaciones mentales así como la diversas interacciones, por lo que la gestión tiene una relación directa con la educación, (UNESCO,

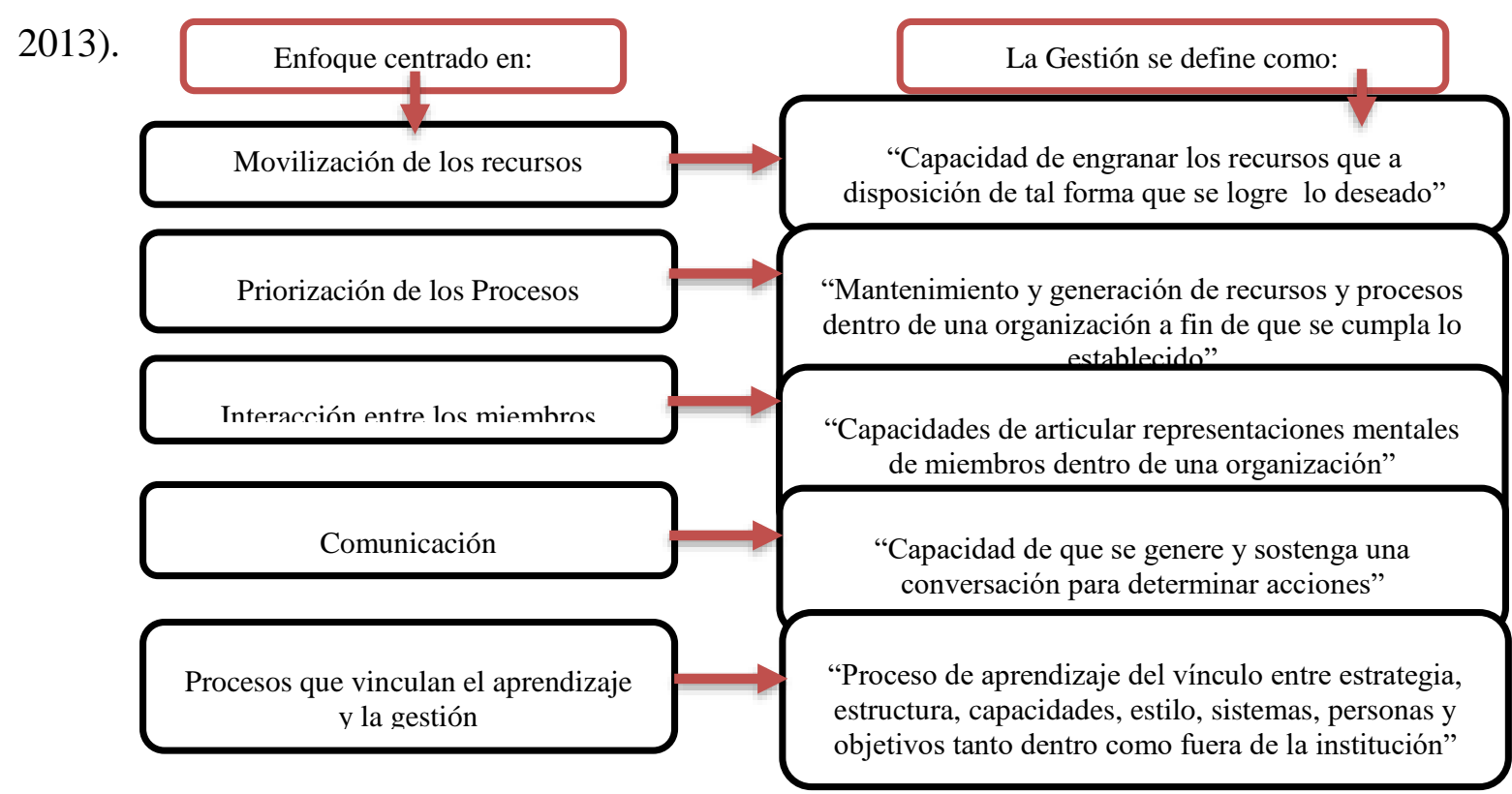

Figura 5: Enfoques de la gestión Fuente: UNESCO (2013). 
Modelos de gestión según Cassasús (2014)

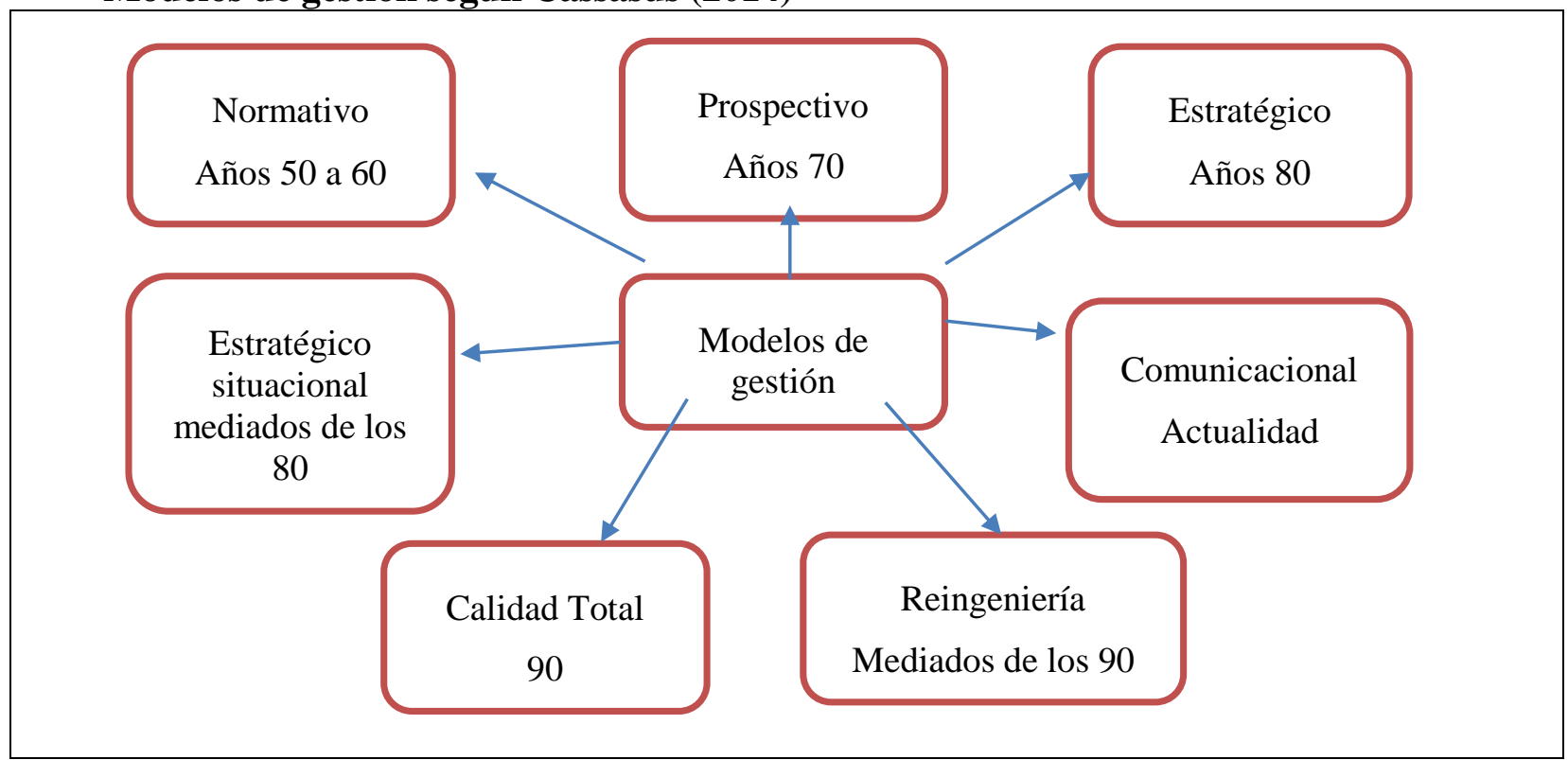

Figura 6: Modelos de gestión según Cassasús (1999). Elaboración propia. Fuente: Casassus (2014).

\section{Calidad de la Tutoría.}

El tutor y la tutoría Según Cruzata (2018) etimológicamente, tutor significa: "maestro, amigo, guía, acompañante, defensor, protector, tutor, director y apoyo". El tutor es un asesor, un mentor, una guía y al mismo tiempo, un supervisor. El trabajo del tutor es crítico porque él / ella debe saber qué y cómo guiar a los estudiantes tutorizados sin sustituirlos el trabajo de los alumnos y lograr su máxima independencia. En otras palabras, para llevar a cabo su trabajo, los tutores deben confiar no solo en el conocimiento del área de estudio correspondiente, pero también en una serie de psicológicos y lineamientos pedagógicos para reforzar su trabajo, especialmente aquellos relacionados con la naturaleza creativa de su trabajo.

Para Sánchez (2015), en una educación abierta y a distancia, un tutor tiene tres funciones: guiar, enseñar y motivar. En el primero, el alumno se introduce al método de estudio, en el segundo, el alumno debe ser garantizó los recursos y condiciones necesarios para la transmisión de conocimiento; y en el tercero, el alumno debe descubrir sus aspiraciones, intereses y motivaciones para poder realizar sus deberes en una de manera más responsable hasta alcanzar los objetivos establecidos e incluso establecer nuevos objetivos cualitativamente superiores

En este sentido, la tutoría actualmente se realiza en varios centros educativos modos, abarca muchos aspectos del saber y en diferentes niveles educativos (pregrado, posgrado), 
entonces lo que se entiende por tutor y la respectiva función son diferentes en temas prácticos.

\section{ESTRATEGIAS METODOLÓGICAS O MATERIALES Y MÉTODOS}

\section{Tipo de estudio}

El presente estudio es de tipo Aplicado, debido a que se tuvo un enfoque donde las teorías se tienen en cuenta para el desarrollo de nuevos conocimientos y que son tratados como tal (Concytec, 2017)

\section{Diseño de investigación}

Correspondiente al diseño de investigación de este estudio es mixto, de tipo descriptivo propositivo, dado que, no se ejecutó ninguna variación de manera intencionada a la variable independiente para determinar los efectos generados en el resto de las variables no comprendidas en el estudio, asimismo, están son descritas de acuerdo a su comportamiento de forma cuantitativa y cualitativa para proponer en base a la deficiencias un nuevo esquema y modelo de comportamiento óptimo dentro de los parámetro de la teoría aplicada.. (Hurtado, 2010, p. 567- 568)

No experimental proyectiva

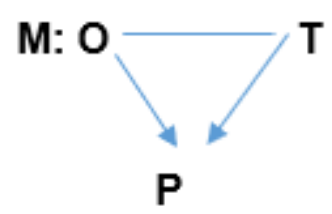

Donde:

$\mathrm{O}$ = Diagnóstico o estado actual de la calidad de la tutoría en la EBR

$\mathrm{T}=$ Teorías referente a calidad de la tutoría y gestión educativa

$\mathrm{P}=$ Propuesta del modelo de gestión educativa.

- Población: En el actual estudio investigativo la población será conformada por:

Población 1: Directivos de la Institución educativa que equivalen a 5

Población 2: Docentes de la institución educativa que equivalen a 82

Población 3: Estudiantes de la institución educativa que equivalen a 1326 alumnos de la educación básica regular.

- Muestra: La muestra en la presente investigación se encuentra conformada por:

Muestra 1: Conformada por el 100\% de la población 1 en estudio, que son 5 directivos.

Muestra 2: Conformada por el 100\% de la población 2 en estudio, que son 82 docentes. 
Muestra 3: Se aplicará la fórmula de muestreo no probabilista de muestreo de población finita, obteniendo 5 grupos de 8 estudiantes por grupos como muestra. Obteniendo 40 alumnos

Objetivo 1. Caracterizar la calidad de la tutoría en la Educación Básica Regular del colegio Francisco Izquierdo Ríos, Morales, San Martín, 2020.

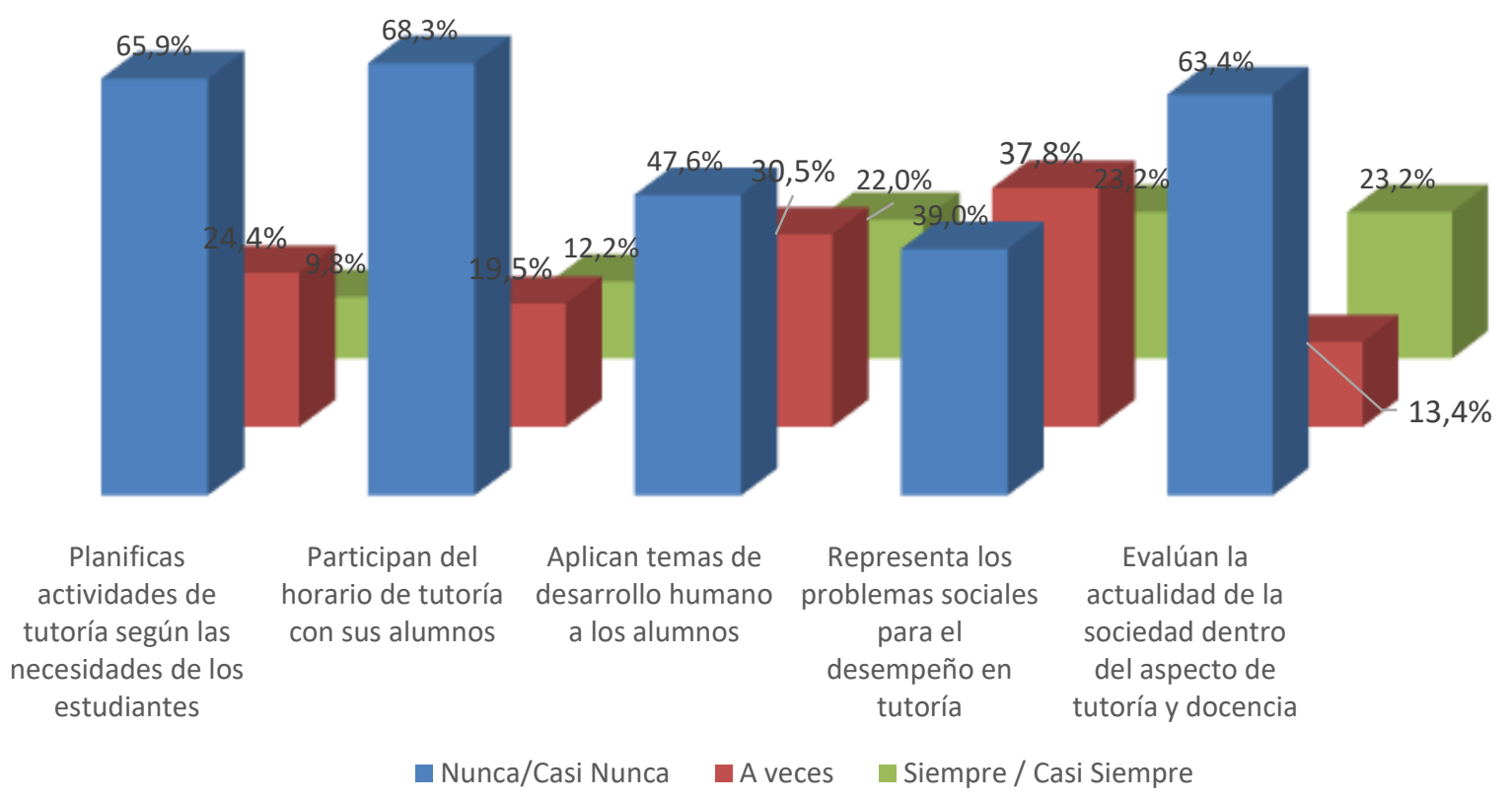

Figura 1. Calidad de la tutoría psicológica desde la percepción del docenteDesarrollo Humano- Fuente: Elaboración Propia

\section{Interpretación}

Del $100 \%$ de los encuestados, referente a la calidad de la tutoría psicológica - Desarrollo humano, el 65.9\% manifiestan que "Nunca o casi nunca" planifican actividades de tutoría según las necesidades de los estudiantes, ni participan del horario de tutoría con sus alumnos (68,3\%). Asimismo, en tanto a la aplicación de temas de desarrollo humano el $47.6 \%$ menciona que no se ejecuta, ni se representan los problemas sociales en el desempeño de la tutoría (39\%), ni se realiza evaluación de la actualidad $(63,4 \%)$. 


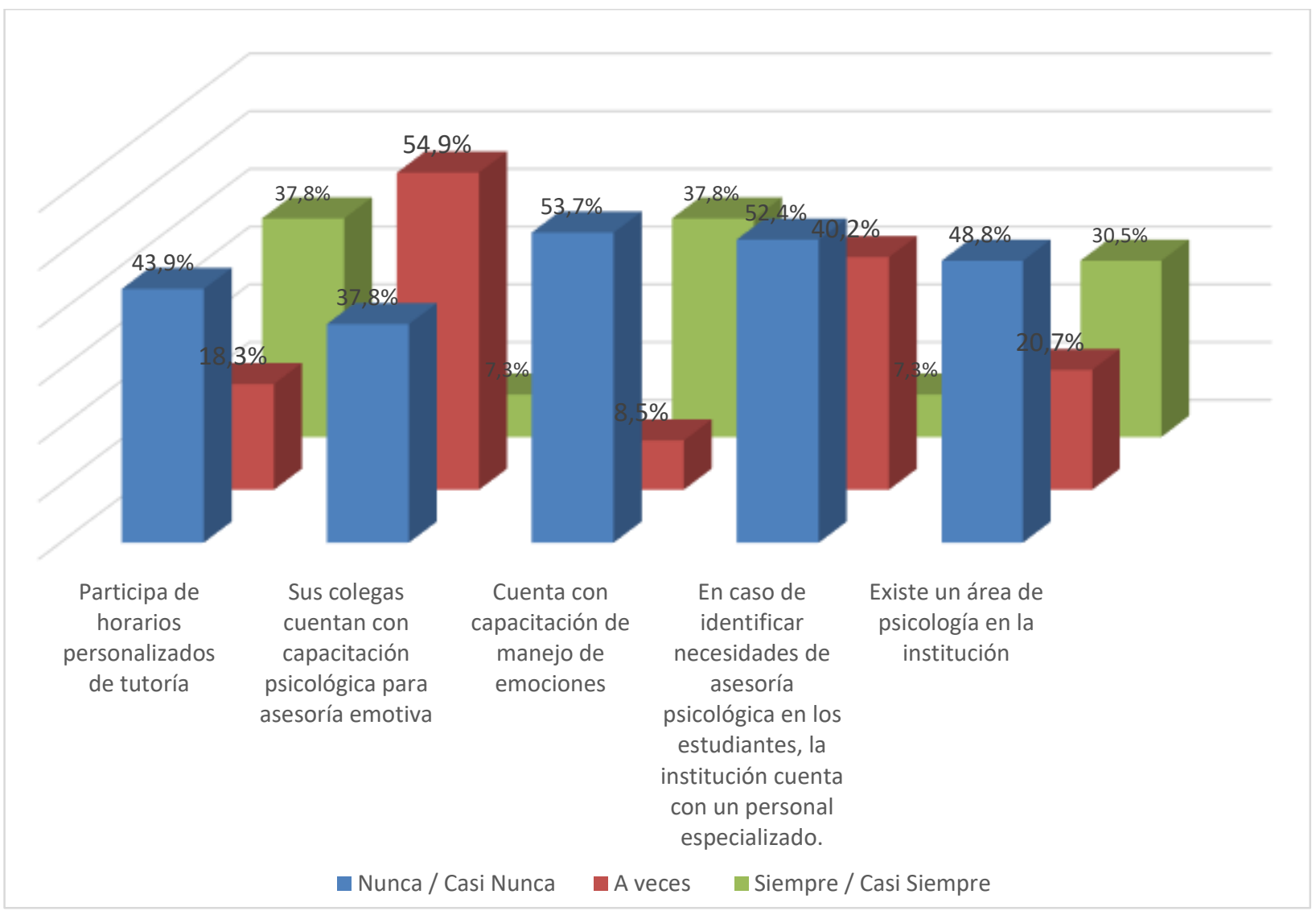

Figura 3. Calidad de la tutoría psicológica desde la percepción del docente - Rol Emotivo del Estudiante -

Fuente: Elaboración Propia

\section{Interpretación}

Del 100\% de los encuestados, referente a la calidad de la tutoría psicológica - Rol emotivo del estudiante, el 53,7\% manifiesta que "Nunca o casi nunca" se capacitan en el manejo de las emociones, no se identifica las necesidades de asesoría $(52,4 \%$,$) , ni participan en$ horarios personalizados como tutor $(43,9 \%)$, tampoco, son capacitados en psicología para asesoría emotiva (37,8\%). Esto podría deberse a que no existe un área de psicología en la institución $(48,8 \%)$. 


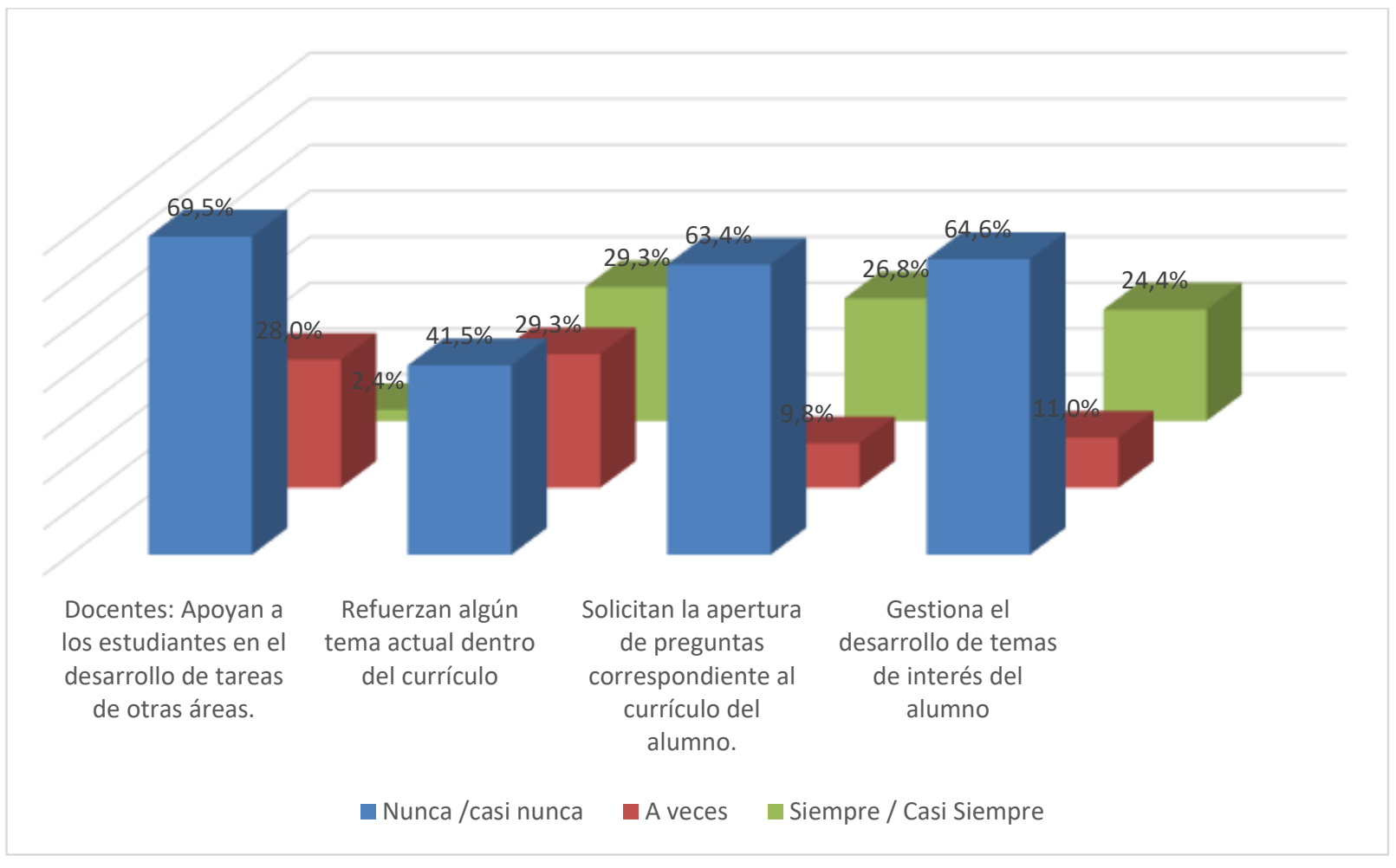

Figura 5. Caracterización de la calidad de la tutoría académica desde la percepción del docente - Rol Curricular.

Fuente: Elaboración Propia

\section{Interpretación}

Del $100 \%$ de los encuestados, referente a la calidad de la tutoría académica - Rol curricular, el 69.5\% manifiestan que "Nunca o casi nunca" apoyan a los estudiantes en el desarrollo de tareas de otras áreas. En cuanto al reforzamiento de algún tema actual dentro del currículo el $29.3 \%$ a veces participan de las mismas.

En tanto a la solicitud de apertura de preguntas correspondiente al currículo el $63.4 \%$ explica que "nunca y casi nunca" aplican el sistema, ni gestionan el desarrollo de temas de interés del alumno en un $64.4 \%$. 


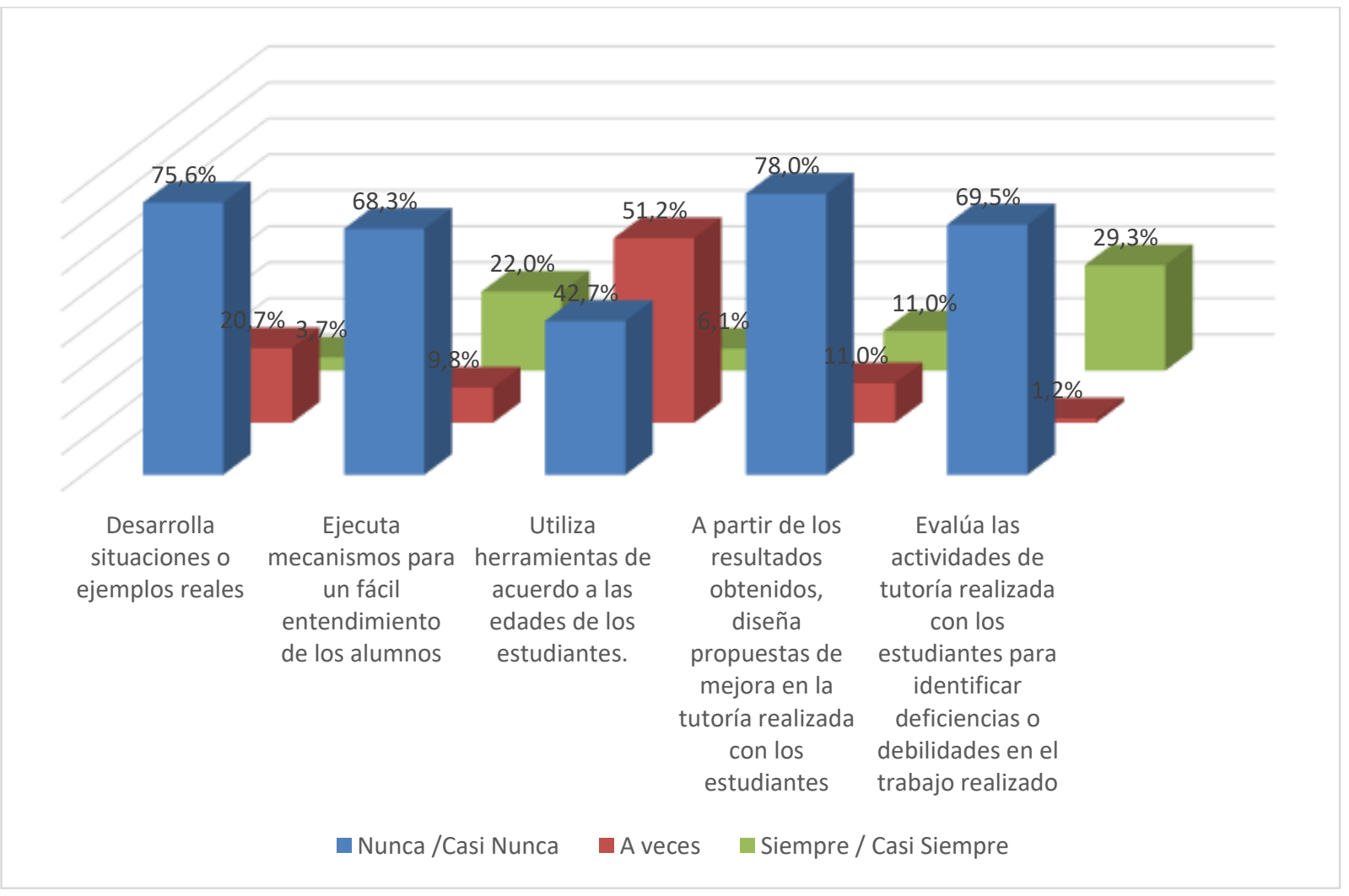

Figura 7. Caracterización de la calidad de la tutoría académica desde la percepción del docente - Asesoría psicológica

Fuente: Elaboración Propia

\section{Interpretación}

Del $100 \%$ de los encuestados, referente a la calidad de la tutoría académica - Asesoría psicológica, el 75.6\% manifiestan que "Nunca o casi nunca" desarrollan situaciones o ejemplos reales. En cuanto a la ejecución de mecanismos para un fácil entendimiento del alumno el $68.3 \%$ menciona que nunca participan.

En tanto a la utilización de herramientas a las edades de los estudiantes el 51.2\% explica que a veces aplican el sistema y 78.0\% nunca diseña propuestas de tutoría para sus alumnos. Y por último el $69.5 \%$ no evalúa las actividades para identificar deficiencias o debilidades del trabajo. 
Objetivo 2. Caracterizar la calidad de la gestión educativa en la Educación Básica Regular del colegio Francisco Izquierdo Ríos, Morales, San Martín, 2020

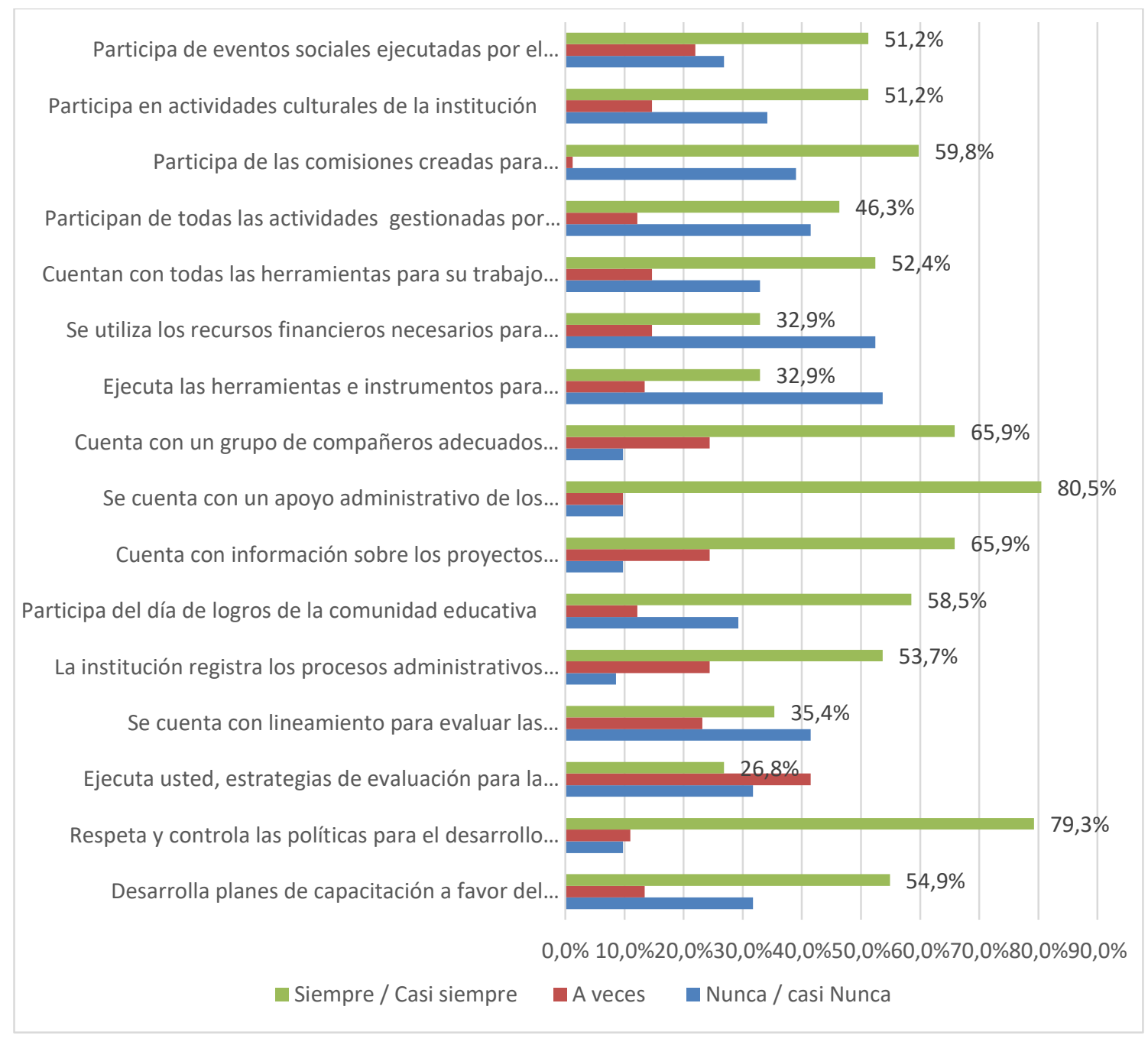

Figura 9. Caracterización de la gestión educativa desde la percepción del docente

Fuente: Elaboración Propia

\section{Interpretación}

Del 100\% de los encuestados, referente a la gestión educativa desde la percepción docente refiere que los directivos cuentan con apoyo administrativo para realizar las gestiones externas $(80,5 \%)$, se respeta y controla las políticas para el desarrollo de competencias de la comunidad educativa (79.3\%), se cuenta con compañeros e información de proyectos que permiten fortalecer la educación (65.9\%), participan en las comisiones creadas para actividades intramuro en un $(59.8 \%)$, desarrollan planes de capacitación para docentes $(54.9 \%)$ y se registra los procesos administrativos incurridos por docentes y/o estudiantes $(53.7 \%)$. 


\section{CONCLUSIÓN O CONSIDERACIONES FINALES}

El papel que tiene las instituciones de educación básica regular para apoyar el tutoría es muy grande ya que deben enfrentar y participar en los diversos problemas académicos que afectan a los estudiantes aportando vivencias y experiencias del profesorado para superarlos y muy específicamente en el colegio Francisco Izquierdo Ríos, es por esto la importancia que tiene la gestión educativa dentro de los procesos de la institución como factor de calidad fortaleciendo las políticas existentes.

Con los resultados de esta investigación se percibir que la mayoría de los entrevistados tienen conocimiento de la gestión educativa y la importancia de la tutoría, sin embargo, un $33 \%$ de los encuestados consideran que debería de incrementarse el nivel de compromiso de la institución como eje rector para la consolidación de la gestión educativa

De acuerdo a nuevo modelo de creación de la gestión educativa de la institución es imprescindible la mejora de la tutoría o parte de su enseñanza y de cómo llegar al estudiante con parámetro establecidos de estudios en base a los 4 pilares de la gestión educativa.

\section{LISTA DE REFERENCIAS}

Arias, F. (2012). El proyecto de investigación (6ta ed.). Venezuela: Episteme. Obtenido de

https://www.researchgate.net/publication/301894369_EL_PROYECTO_DE_IN VESTIGACION_6a_EDICION

Bernal, C. (2016). Metología de la investigación (4ta ed.). Colombia: Pearson.

Buitrago, G. (2015). Estrategia de gestión escolar para la prevención del consumo de drogas en adolescentes de la básica y la media del colegio Costa Rica IED Bogotá DC. Bogotá, Colombia: Universidad Libre. Obtenido de http://repository.unilibre.edu.co/bitstream/handle/10901/8339/ESTRATEGIA\% 20DE\%20GESTI\%C3\%93N\%20ESCOLAR\%20PARA\%20LA\%20PREVENCI $\%$ C3\%93N\%20DE\%20LA\%20DROGADICCI\%C3\%93N.pdf?sequence=1

Cassasús, J. (2014). La gestión educativa en América Latina. Perú: Micentroeducativo. Obtenido de http://www.micentroeducativo.pe/2014/docente/fileproject/file_docentes/91bi_7 7e3a9.pdf 
Chipana, M. (2015). Gestión pedadgógica y calidad educativa en las unidades de gestion educativa local San Román Azángaro - 2013. Juliaca, Perú: Universidad Andina "Néstor Cáceres Velásquez". Obtenido de http://repositorio.uancv.edu.pe:8080/bitstream/handle/UANCV/276/TESIS.pdf? sequence $=1 \&$ isAllowed $=\mathrm{y}$

Coronado, M. (2017). Tutoría y orientación educativa para mejorar las habilidades sociales en los etudiantes de la institución educativa Multigrado $\mathrm{N}^{\circ} 16165$, Huaranguillo - San José del Alto - Jaén. Cajamarca, Perú: Universidad nacional de Cajamarca. Obtenido de http://repositorio.unc.edu.pe/bitstream/handle/UNC/1098/TUTOR\%C3\%8DA\% 20Y\%20ORIENTACI\%C3\%93N\%20EDUCATIVA\%20PARA\%20MEJORAR \%20LAS\%20HABILIDADES\%20SOCIALES\%20EN\%20LOS\%20ESTUDIA NTES\%20DE\%20LA\%20IN.pdf?sequence=1\&isAllowed=y

Delgado, E.; Ravello, C.; Ramos, M. ; Sabaduche, L. \& Flores, C. (2007). Tutoría y orientación educativa en la educación primaria. LIma, Perú: MINEDU. Obtenido de http://tutoria.minedu.gob.pe/assets/tutoria-y-orientacion-educativa-en-laeducacion-primaria.pdf

Díaz, D \& Delgado, M. (2014). Competencias gerenciales para los directivos de las instituciones educativas de Chiclayo: Una propuesta desde la socioformación. Chiclayo, Perú: Universidad Católica Santo Toribio de Mogrovejo. Obtenido de http://tesis.usat.edu.pe/bitstream/usat/563/1/TM_DiazCastilloDoris_DelgadoLey vaMiguel.pdf

Domínguez, G.; Domínguez, A. \& Torres, J. (2016). Didáctica y aplicación de la administración de operaciones. México: IMCP. Obtenido de https://books.google.com.pe/books?id=Zud0DgAAQBAJ\&pg=PA109\&dq=cicl o+de+deming + pdf \&hl=es419\&sa=X\&ved=0ahUKEwjp_pfKvt7ZAhVkkeAKHRx9AEwQ6AEITTAH\#v $=$ onepage $\& \mathrm{q}=$ ciclo $\% 20 \mathrm{de} \% 20 \mathrm{deming} \% 20 \mathrm{pdf} \& \mathrm{f}=$ false 
Fiallos, M. (febrero de 14 de 2014). Los valores y el rendimiento escolar. El nuevo diario, pág. 2014. Obtenido de https://www.elnuevodiario.com.ni/opinion/311112valores-rendimiento-escolar/

Flores, V. (2012). Influencia significativa del programa de tutoria y orientación Educativa-Toe en la eficacia del docente tutor del nivel secundaria de las instituciones educativas de la unidad de gestión educativa local UGEL 04 Comas. Año 2009. Lima, Perú: Universidad Mayor de San Marcos. Obtenido de http://cybertesis.unmsm.edu.pe/bitstream/handle/cybertesis/925/Flores_lv.pdf;js essionid=F2ADE982A6F4AC6BF63175929469C087? sequence $=1$

Frigerio, G.; Poggi, M. ; Tiramonti, G.; Aguerrondo, I. (1992). Las instituciones educativas Cara y Seca. Buenos Aires, Argentina: Torquel. Obtenido de https://es.scribd.com/document/356016498/Frigerio-Graciela-Las-InstitucionesEducativas-Cara-y-Ceca-pdf

Hurtado, A. (2015). Planificación estratégica como herramienta para el fortalecimiento de la gestion educativa en la escuela técnica "Simón Bolívar" Nicaragua estado Carabobo. Carabobo, Venezuela : Universidad de Carabobo. Obtenido de http://riuc.bc.uc.edu.ve/bitstream/123456789/913/1/A.Hurtado.pdf

Hurtado, J. (2010). Metodología de la investigación. Bogotá, Colombia: Quirón.

Hurtado, N. \& Lancheros, R. (2016). Estrategias de gestión educativa para fortalecer la convivencia en estudiantes de ciclo I y II del colegio Orlando Higuita, Rojas IED. Bogotá, Colombia: Universidad Libre. Obtenido de http://repository.unilibre.edu.co/bitstream/handle/10901/8274/Estrategia\%20de $\% 20$ gesti\%C3\%B3n\%20educativa\%20para\%20fortalecer\%20la\%20convivencia $\% 20$ en $\% 20$ estudiantes $\% 20 \mathrm{de} \% 20$ ciclo $\% 20 \mathrm{I} \% 20 \mathrm{y} \% 20 \mathrm{II} \% 20 \mathrm{del}$.pdf?sequence $=1$

Icahuate, A. \& Huansi, D. (2015). Diseño de una estrategia de gestión educativa para mejorar los niveles de convivencia en la institución educativa Túpac Amaru, 2013. Iquitos, Perú: Universidad nacional de la Amazonía Peruana. Obtenido de http://repositorio.unapiquitos.edu.pe/bitstream/handle/UNAP/3731/Lizeth_Tesis _Maestria_2015.pdf?sequence=1\&isAllowed =y 
Iguíñiz, M. (2017). Opinión: El proyecto educativo nacional al 2021 y la situación actual. Perú: Edugestores. Obtenido de http://www.edugestores.pe/opinion-el-proyectoeducativo-nacional-al-2021-y-la-situacion-actual-por-manuel-iguiniz/

Ley General de la Educación N 28044. (2014). Lima, Perú: Minedu. Obtenido de http://www.minedu.gob.pe/p/ley_general_de_educacion_28044.pdf

Ministerio de educación. (2014). Dirección de Tutoría y Orientación Educativa. Perú: MINEDU. Obtenido de http://www.minedu.gob.pe/ditoe/

Miranda, C. (2015). El sistema de tutoría en el desarrollo integral de los estudiantes de la Universidad César Vallejo Chiclayo. Lambayeque, Perú: Universidad César Vallejo. Obtenido de http://repositorio.ucv.edu.pe/bitstream/handle/UCV/7425/Miranda_JCDLM.pdf? sequence $=4 \&$ isAllowed $=\mathrm{y}$

Muñoz, C. (2013) " Principales problemas que afectan al desarrollo del sistema educativo". Investigador del Instituto de Investigaciones para el Desarrollo de la Educación (INIDE) de la Universidad Iberoamericana (UIA). Especialista en temas de planeación educativa; educación, economía y sistema productivo. Premio Nacional de Ciencias y Artes

Niño, M.; Mendoza, F. \& Méndez, J. (2013). Tutoría y orientación educativa en el nivel superior desde la perspectiva de la organización escolar (Vol. 10). México: Pepsic. Obtenido de http://pepsic.bvsalud.org/scielo.php?script=sci_arttext\&pid=S166575272013000200010

Noreña, A.; Alcaraz N.; Rojas, J. \& Rebolledo, D. (2012). Aplicabilidad de los criterios de rigor y éticos en la investigación. Aquichan, 263-274. Obtenido de http://www.redalyc.org/articulo.oa?id=74124948006

Ñaupas, H.; Mejía, E.; Novoa, E. \& Villagómez, A. (2014). Metodología de la investigación. Bogotá, Colombia |: De la U.

Ravello, C. ; Martínez, C \& Delgado, G. (2007). Manual de Tutoría y Orientación Educativa. Lima, Perú: Quebecor. Obtenido de 
http://www.minedu.gob.pe/minedu/archivos/a/002/02-bibliografia-comun-a-ebreba-y-etp/3-manual-de-tutoria-y-orientacion-educativa.pdf

Rojas, N. \& Gonzáles, I. (2015). Estrategia de gestión educativa para transformar la convivencia en el colegio eduardo Umaña de la localidad de Kennedy. Bogotá, Colombia: Universidad Libre.

Sáez, R. (15 de Octubre de 2015). ¿Cuáles son las razones del fracaso escolar de nuestros hijos? Hola.com. Obtenido de https://www.hola.com/ninos/2015101581540/fracaso-escolar-causas-ysoluciones/

Saravia, G. (2018). Gestión educativa y la calidad en la formación tecnológica del instituto superior Público San Francisco de Asis Chincha, 2017. Lima, Perú: Universidad César Vallejo. Obtenido de http://repositorio.ucv.edu.pe/bitstream/handle/UCV/10781/Saravia_RGP.pdf?se quence $=1 \&$ is Allowed $=\mathrm{y}$

Soto, J. (14 de diciembre de 2017). No entiendo la escuela sin educar en valores. La voz de Galicia.

UNESCO. (2011). Manual de gestión para directores de instituciones educativas. Lima, Perú: $\quad$ MInedu. Obtenido de http://unesdoc.unesco.org/images/0021/002191/219162s.pdf 